\title{
Nutrition support practices in South African ICUs: Results from a nationwide pilot survey
}

\author{
L T Hill, PhD RD (SA) \\ Critical Point Critical Care Nutrition Consultancy, Cape Town, South Africa
}

Corresponding author: L T Hill (laurenhill@mweb.co.za)

Background. Nutrition support of the critically ill can positively affect clinical outcomes. International audit data of nutrition practices in intensive care units (ICUs) suggest that inconsistent application of recommended nutrition support practices (NSPs) occurs. There are no data on NSPs in South African (SA) ICUs.

Objective. To perform a national pilot survey of NSPs in private SA adult ICUs.

Methods. A descriptive, observational, cross-sectional survey was performed among prescribers of nutrition support in private ICU facilities. Participants were targeted through non-randomised convenience sampling and invited to complete a 51-item electronic questionnaire covering ICU demographics, profile of nutrition prescribers, and nutrition assessment support, delivery and monitoring practices.

Results. Responses were received from 125 practitioners in $60 \%$ of representative private hospitals with ICU facilities, mainly general/ mixed ICUs. Forty-six percent of respondents reported structured nutrition support teams and $61 \%$ reported that practices were governed by formal nutrition support protocols. Enteral nutrition was reported to be based upon published guidelines by $72 \%$ of dietitians, while parenteral nutrition decisions were reportedly based mainly on clinical judgement (43\%). For both enteral and parenteral feeding practices, compliance with guidelines was inconsistent. There was a disjuncture between various NSPs as reported by dietitians and by nurses. Nurses generally appeared unaware of published nutrition guidelines.

Conclusion. Various disparities in reported nutrition practices were revealed, suggesting that the organisation and operations of teams and the implementation of protocols informed by published nutrition guidelines may not be well established in private SA ICUs.

S Afr J Crit Care 2015;31(2):42-50. DOI:10.7196/SAJCC.2015.v31i2.252

Nutrition support of a critically ill patient is a form of therapy ${ }^{[1]}$ and positively affects important clinical outcomes. Various studies have shown that it reduces infectious morbidity, duration of mechanical ventilation, length of stay, impaired functional capacity and the overall cost of care. ${ }^{[2-6]}$ Despite this evidence, prevalence of malnutrition in intensive care unit (ICU) patients remains high. ${ }^{[7,8]}$ This suggests that nutrition support practices (NSPs) are still suboptimal in many clinical settings.

Globally, information about NSPs in ICUs is scarce. An extensive survey of NPSs in 20 European countries ${ }^{(9)}$ indicated that NSPs and, in particular, consistent adherence to recommended practices, varied widely throughout the countries surveyed. No data are available on factors related to NSPs in South African (SA) ICUs, which may differ from other parts of the world. In addition, most ICU practice occurs in the private healthcare sector, which is largely run using an open-unit model without a full-time intensivist who maintains executive clinical oversight of all aspects of patient care. Previous SA audit data indicate that $4 \%$ of private ICUs were compliant with the closed-unit model. ${ }^{[10]}$ While closed units are reportedly an effective approach to enhanced clinical outcomes owing to integrated care, ${ }^{[10]}$ the private sector is subject to minimal constraints on resources, which may also contribute differently to quality of care.

Furthermore, the typical SA ICU patient differs from that of developed countries in ways that are of relevance to NSPs; patients are generally younger, more likely to enter ICU as a result of trauma, and beset with a different underlying disease burden, most notably HIV and tuberculosis (TB). ${ }^{[11,12]}$

These factors may all influence the manner in which nutrition support is utilised and delivered in SA ICUs, and whether published international nutrition practice guidelines are being followed.
Given these factors and the lack of SA data, the objective of this study was to perform the first national survey of nutrition support and related practices in SA ICUs within the private healthcare structure.

\section{Methods}

This was a descriptive, observational, cross-sectional survey of nutrition practices of ICU dietitians, doctors and nursing managers working daily in private SA adult ICUs. The study utilised a non-randomised convenience sample of healthcare practitioners practising in the 154 private hospitals with adult ICUs in SA, with the objective to obtain a response from at least one key nutrition prescriber per adult ICU nationwide. Target participants were invited to complete a 51-item online questionnaire covering: (i) ICU and patient demographic characteristics; (ii) professional profile of ICU nutrition support prescribers; (iii) nutrition screening and assessment practices; and (iv) nutrition support prescription, delivery and monitoring practices. Participants were also invited to share anonymised written nutrition protocols. The electronic survey was managed via a professional online survey company that provided electronic announcements, reminders and unique invitation links to the survey instrument to targeted participants, as well as an open link for any additional responders, and returned anonymised, uncleaned but collated data to the researchers.

Ethical approval to perform the study was obtained from PharmaEthics Independent Research Ethics Committee (ref. number: 150110873), the Research and Ethics Committees of the private hospital groups, the applicable regional managers of the private hospital groups, and nursing managers of individual hosptials. Survey completion was voluntary and data were anonymised.

Statistical analysis was done using Statistica 12 (Statsoft, USA). Results are presented as mean (standard deviation (SD)) or median (interquartile 
range (IQR)) for continuous data, depending on the normality of data. Categorical data are described as frequencies or percentages. Group differences in frequencies were analysed using Fisher's exact test for small samples. In such cases $p<0.05$ was taken as statistically significant. Owing to the very small number of doctor responders, this group was combined with dietitians for subgroup analyses.

\section{Results \\ Sample and ICU demographics}

A total of 961 targeted electronic survey invites were sent to all 154 private hospitals with adult ICUs, and there were 125 (13\%) responses. The responses came from 93 distinct ICUs, representing $60 \%$ of the private adult ICUs in the country, but were concentrated in the three large metropole areas of the Western Cape, Gauteng and KwaZuluNatal provinces. In addition, there were seven responses from unidentified hospitals. Responses from the nine SA provinces reflected the proportional regional distribution of ICUs throughout the country, indicating that this pilot survey is nationally representative of all the private adult ICU facilities nationwide (Fig. 1). The ICU capacity was reported to be a median (IQR) of $15(12-26)$ beds (Table 1$)$. Most practitioners reported working mainly in general/mixed ICUs (85\%) with mixed gender. The majority of responders (67\%) were dedicated to work in a single ICU, while the remaining respondents reported clinical coverage of between two and seven separate ICUs. Surveys were completed by dietitians (48\%), ICU nursing managers (48\%), intensivists (2\%) and specialist physicians (2\%). Only one participant shared a nutrition protocol with the researcher.

\section{Clinical structure for nutrition support}

Overall, $46 \%$ of respondents reported the presence of a formal nutrition support team in the ICU. However, significantly more ICU nursing managers (56\%) reported the presence of a nutrition support team compared with reports from dietitians and doctors combined (37\%, $p=0.03)$. Questions regarding professional composition of the nutrition support team yielded disparate results. Overall, $26 \%$ of respondents indicated that the nutrition support team comprised a dietitian, nurse, doctor and pharmacist, $19 \%$ by a dietitian and doctor, and $16 \%$ by a dietitian, nurse and doctor. However, 31\% of respondents reported that the nutrition support team comprised a dietitian acting alone in managing nutrition support in the ICU, and $41 \%$ of overall responses included the ICU nurse as part of the team. For responses from the ICU nursing manager subgroup, only $33 \%$ included the ICU nurse as in the nutrition support team, while $42 \%$ viewed the dietitian acting alone as the nutrition support team. In contrast, $58 \%$ of dietitians included nurses in the team. No doctors included nurses in the professional composition of the nutrition support team. Only $61 \%$ of ICUs reported the presence of formal, written nutrition support protocols governing nutrition support. Of those, 54\% covered both enteral and parenteral nutrition support, while $41 \%$ only governed enteral nutrition support and the remainder covered only parenteral nutrition support.

\section{NSPs}

\section{Nutrition assessment}

Overall, $82 \%$ of ICUs reported that nutrition assessment of patients was routine, with $83 \%$ identifying the dietitian as responsible for this task and the remaining identifying either the nurse or doctor, or a joint effort. However, $54 \%$ omitted to answer this question. Nutrition assessment was reported by $68 \%$ of dietitians

\section{Table 1. Profile of responding ICUs and patients $(N=125)$}

\begin{tabular}{lll}
\hline ICU type & $\boldsymbol{n}(\%)^{*}$ & Bed capacity, mean (SD) \\
\hline General/mixed & $106(85)$ & $14.3(7.9)$ \\
Surgical & $31(25)$ & $11.9(6.4)$ \\
Medical & $24(19)$ & $13.7(5.8)$ \\
Cardiothoracic & $33(26)$ & $12.3(6.2)$ \\
Neurological & $13(10)$ & $8(1-8)^{\dagger}$ \\
Burns & $6(5)$ & $14(8-30)^{\dagger}$ \\
Trauma & $15(12)$ & $6(6-6)^{\dagger}$ \\
Overall median & & $15(12-26)^{\dagger}$ \\
Primary diagnosis & $n(\%)^{*}$ \\
\hline Mainly elective surgery & $34(27)$ \\
Mainly emergency, non-trauma surgery & $9(7)$ & \\
Mainly medical, including obstetrics & $35(28)$ \\
Mainly mixed trauma & $20(16)$ \\
A mix of elective and emergency surgery & $20(16)$ \\
Mainly cardiac/cardiothoracic & $6(5)$ \\
${ }^{+}$Mesponses exceed 100\% as multiple choices were possible. & \\
\hline
\end{tabular}

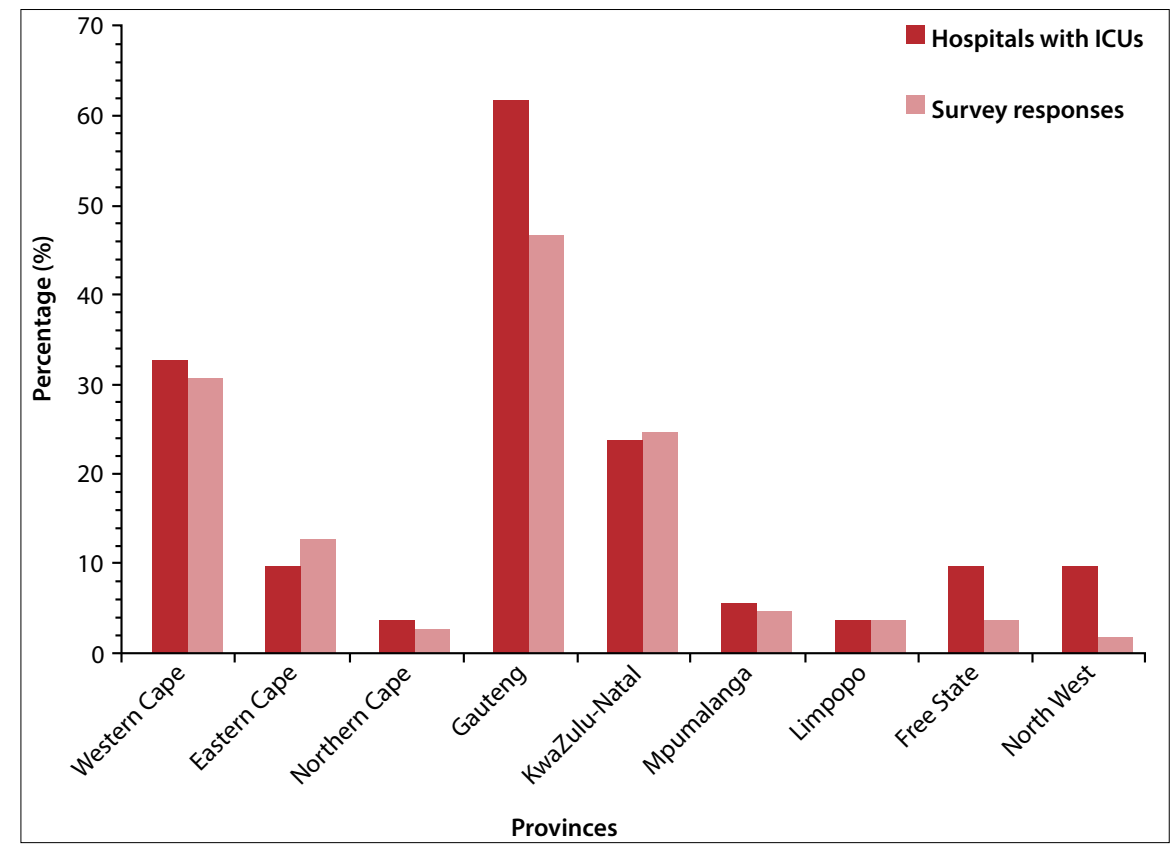

Fig. 1. National representation of ICU facilities. 
as being achieved by a combined $A B C D$ (anthropometry, biochemistry, clinical, dietary) approach, while the remainder reported to use various other standard nutrition assessment tools in combination. For subgroup responses, $48 \%$ of nurses and $50 \%$ of the doctors $(n=2)$ were unsure of the nutrition assessment method used in the ICU.

\section{Nutrition decision-making}

Summary data for nutrition decision-making practices are shown in Table 2 and Fig. 2. The primary decisions about route of nutrition delivery were made jointly by dietitians and doctors (57\%), followed by doctors alone (34\%). The group overall and the two profession subgroups separately indicated that nursing involvement in this decision was low (3\%), although nurses were included in decisionmaking about daily fluid volumes allocated to nutrition in most responses (51\%). The dietitians and doctors subgroup reported using published guidelines (European Society for Parenteral and Enteral Nutrition (ESPEN), American Society for Parenteral and Enteral Nutrition (ASPEN), Canadian or other) to determine nutrition requirements in $21 \%$ of responses, with $62 \%$ reporting the use of the above-mentioned guidelines together with equation-based and other methods. The remaining responses reported the use of body weight-based methods (8\%), and equations such as Harris-Benedict (8\%) without reference to guidelines. Of nurses, $42 \%$ responded that they were unsure of how nutrition requirements were calculated in the ICU, while $15 \%$ reported that body weight-based methods were used and a further $13.5 \%$ reported that requirements were based on clinical judgement. These responses were significantly different between nurses and dietitians and doctors combined (Table 2).

The most commonly reported determinant of enteral feed initiation was haemodynamic stability (43\%) followed by the first ICU day if no

Table 2. Nutrition support decision-making practices

\begin{tabular}{|c|c|c|c|c|}
\hline & $\begin{array}{l}\text { Total group, } \\
n(\%)\end{array}$ & $\begin{array}{l}\text { Dietitians and doctors, } \\
n(\%)\end{array}$ & $\begin{array}{l}\text { Nurses, } \\
n(\%)\end{array}$ & $\begin{array}{l}\text { Intergroup } \\
p \text {-value* }\end{array}$ \\
\hline Decisions regarding route of nutrition support, $N$ & 115 & 63 & 52 & \\
\hline Made by dietitian and doctor together & $66(57)$ & $39(63)$ & $27(52)$ & \\
\hline Made by specialist doctor alone & $39(34)$ & $19(31)$ & $20(40)$ & \\
\hline Made by dietitian alone & $6(5)$ & $3(5)$ & $3(6)$ & \\
\hline Made jointly by nurses and doctors & $3(3)$ & $2(3)$ & $1(2)$ & \\
\hline Methods of calculating nutrition requirements, $N$ & 115 & 63 & 52 & \\
\hline ESPEN/ASPEN or other published guidelines & $15(31)$ & $13(21)$ & $2(4)$ & $<0.050$ \\
\hline Clinical judgement & $7(6)$ & $0(0)$ & $7(14)$ & 0.003 \\
\hline Body weight-based methods & $13(11)$ & $5(8)$ & $8(15)$ & \\
\hline Equations & $7(6)$ & $5(8)$ & $2(4)$ & \\
\hline Indirect calorimetry & $2(2)$ & $0(0)$ & $2(4)$ & \\
\hline Other methods combined with guidelines & $48(42)$ & $39(62)$ & $9(17)$ & \\
\hline Don't know & $23(20)$ & $1(2)$ & $22(42)$ & \\
\hline Fluid volume allocated to nutrition support, $N$ & 113 & 61 & 52 & \\
\hline Decided jointly by multidisciplinary team & $58(51)$ & $30(48)$ & $28(54)$ & \\
\hline Decided by doctor & $49(43)$ & $29(46)$ & $20(38)$ & \\
\hline Decided by dietitian & $6(5)$ & $2(3)$ & $4(8)$ & \\
\hline Determinants of enteral feed initiation, $N$ & 114 & 62 & 52 & \\
\hline First day in ICU & $10(9)$ & $2(3)$ & $8(15)$ & 0.040 \\
\hline Gastric residual volume & $3(3)$ & $1(1.6)$ & $2(4)$ & \\
\hline Haemodynamic stability & $49(43)$ & $31(50)$ & $18(35)$ & \\
\hline Reasonable gastrointestinal function & $21(18)$ & $8(13)$ & $13(25)$ & \\
\hline Presence of bowel sounds & $0(0)$ & $0(0)$ & $0(0)$ & \\
\hline Reasonable nutritional status & $2(2)$ & $1(1.6)$ & $1(2)$ & \\
\hline First day in ICU if no clinical contraindication & $30(26)$ & $19(31)$ & $11(21)$ & \\
\hline Determinants of parenteral feed initiation, $N$ & 114 & 62 & 52 & \\
\hline First day in ICU & $2(2)$ & $0(0)$ & $2(4)$ & \\
\hline Gastrointestinal failure, ileus or obstruction & $2(2)$ & $1(2)$ & $1(2)$ & \\
\hline Any gastrointestinal surgery & $2(2)$ & $0(0)$ & $2(4)$ & \\
\hline Poor nutritional status of patient & $2(2)$ & $0(0)$ & $2(4)$ & \\
\hline Unsuccessful enteral nutrition & $8(7)$ & $4(6)$ & $4(8)$ & \\
\hline GIT factors making EN success unlikely & $89(78)$ & $51(82)$ & $38(73)$ & \\
\hline Other factors & $9(8)$ & $6(10)$ & $3(6)$ & \\
\hline
\end{tabular}


clinical contraindications were evident (26\%). However, $18 \%$ of the respondents overall and $25 \%$ of nurses indicated that enteral feed initiation was determined by reasonable gastrointestinal function. The majority (78\%) of respondents identified the main determinant of parenteral nutrition initiation as the presence of gastrointestinal factors likely to make enteral feeding unsuccessful, while only $7 \%$ indicated this factor to be actually unsuccessful enteral feeding.

The respondents overall and the dietitian and doctor subgroup reported that enteral decisions were governed by a combination of published guidelines and clinical judgement (Fig. 2A). This was significantly different from the nurses' response, which was that the enteral support decision was based on a multidisciplinary team decision. A total of $23 \%$ of respondents reported that enteral nutrition decisions were based solely on the clinical judgement of the dietitian or doctor. In contrast (Fig. 2B), while the majority of dietitians and doctors reported basing parenteral nutrition decisions on published guidelines $(58 \%), 43 \%$ of respondents overall identified clinical judgement as the main factor underlying decisions around parenteral nutrition support. This proportional difference was due to nurses (46\%) perceiving a high reliance on clinical judgement for parenteral decisions, while $0 \%$ of nursing staff reported published guidelines as a basis for parenteral decision-making.

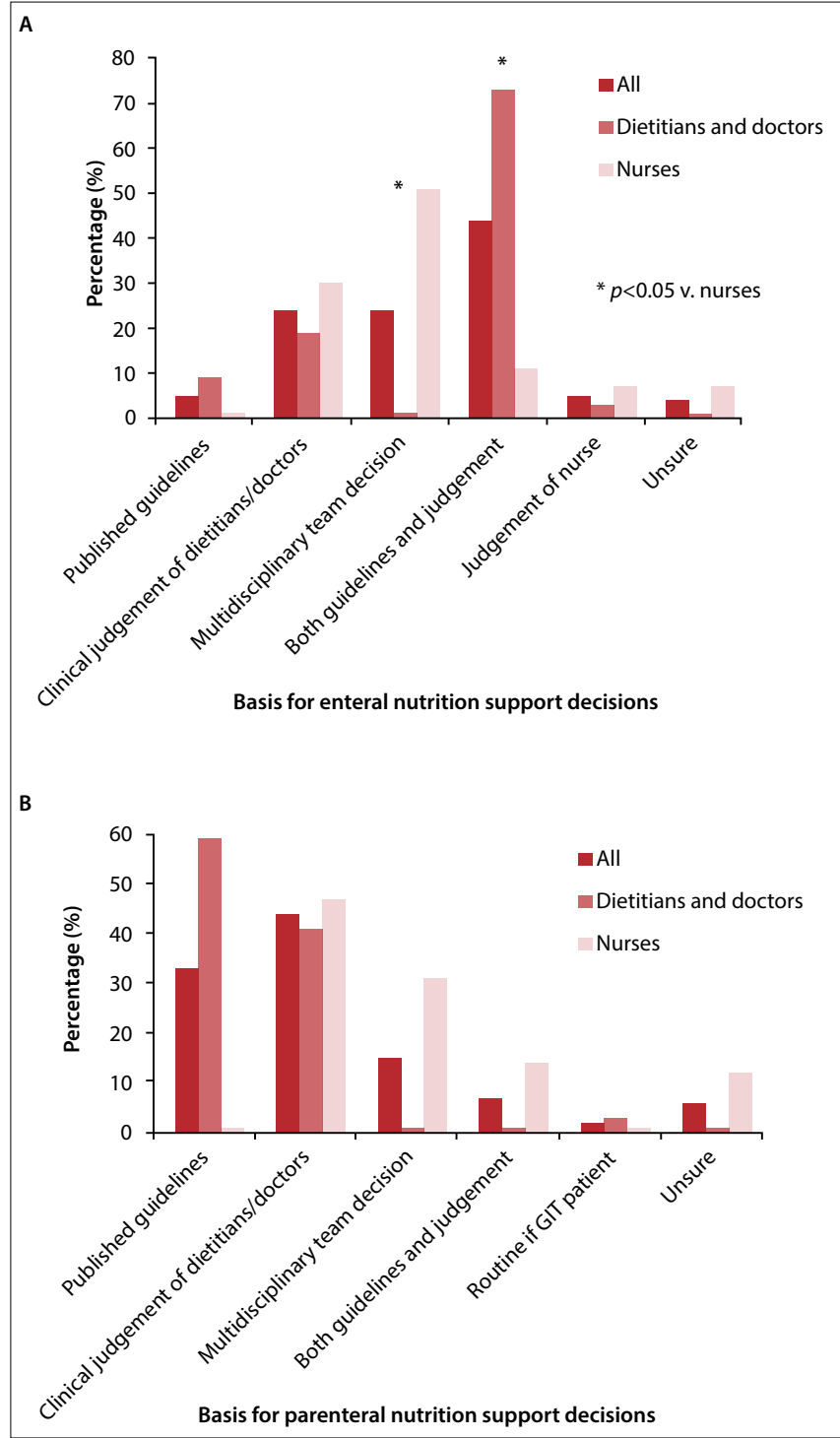

Fig. 2. Basis for enteral and parenteral nutrition support decisions. (GIT = gastrointestinal tract.)

\section{Enteral nutrition delivery, administration and monitoring}

Summary data for enteral support practices are shown in Table 3. Healthcare professions were congruent in reports that enteral feeding was commenced within 24 hours $(47 \%)$ or 48 hours (22\%) of ICU admission. However, $19 \%$ of nursing staffreported that timing of enteral feed initiation was dependent on the practice of individual dietitians or doctors. The nasogastric route of delivery was most commonly used (96\%). While $41 \%$ reported that postpyloric feeding was seldom used, there was a $42 \%$ overall reported use of this route in patients having undergone gastric surgery - although significantly more nurses than dietitians and doctors reported this practice ( $55 \%$ v. $31 \%, p=0.020$ ).

Feeding tube placement depended largely on the dietitian/doctor $(82 \%)$. While dietitians and doctors reported that the most common method of checking tube position was by chest X-ray (49\%), nursing staff reported significantly different bedside methods including use of litmus paper ( $24.5 \%, p=0.000$ v. $5.0 \%$ for dietitians and doctors) and air auscultation ( $41.5 \%, p=0.000$ v. $6.6 \%$ for dietitians and doctors). Pattern of feed delivery was significantly different between dietitians and nurses, with the former reporting mainly 24-hour continuous delivery ( $59 \%$ v. $30 \%, p=0.010$ ) and nurses reporting mainly continuous delivery with short holds ( $45 \%$ v. $34 \%, p=0.020$ ). Again, $13 \%$ of nurses indicated that delivery routine differed for individual doctors and dietitians. Other feed management practices (tube and giving set changes, and tolerance checks) were reported significantly differently between dietitians and doctors and nurses (Table 3). For a number of aspects, $30-40 \%$ of dietitians and doctors responded that they were unsure of the practice.

There were discrepancies in the reported utility of gastric residual volume (GRV) in guiding enteral feeding with a mismatch between $100 \%$ of dietitians and doctors who said that GRV was not used at all to guide practice, while $23 \%$ later reported that it was used alone or in combination with symptoms (57\%) to monitor enteral feed tolerance. In addition, $92 \%$ of nurses reported that GRV was the main method used to check enteral feed tolerance. The question on GRV guidance of practice was answered by a disproportionately low proportion of respondents and was not answered by any respondent who had previously indicated that NSPs were governed by formal nutrition protocols in their ICU. No respondents who had indicated enteral feeding protocol use identified a specific GRV threshold that guided practice.

Enteral feed optimisation methods were different between nurses and the combined dietitians and doctors group, but the total research group overall reported using gastric acid suppression and prokinetic agents when clinically indicated (35\%) or at the request of nursing staff (21\%), while changing the enteral feed was the next most commonly used strategy (19\%). Frequency and method of enteral feed monitoring was different between dietitians and doctors compared with nurses, with $12.5 \%$ of nurses being unsure of methods used.

\section{Parenteral nutrition delivery, administration and monitoring}

There was a low initiation of early parenteral nutrition in malnourished patients (7\%), and clinical indication overrode guideline-based timing according to most responses (30\%) (Table 4). However, $20 \%$ overall and $26 \%$ of nurses reported parenteral nutrition typically started within 24 hours of ICU admission. The majority (87\%) of dietitians and doctors viewed the rationale for parenteral nutrition as the provision of requirements only with unsuccessful enteral support, which was proportionally higher than the percentage of nurses (61\%) with the same view (group difference, $p=0.0065$ ). Relatively more nurses (19.5\%) than dietititians and doctors reported use of parenteral nutrition as a supplement to enteral feeds $(p=0.030)$. Frequency and methods 


\begin{tabular}{|c|c|c|c|c|}
\hline & Total group, $\boldsymbol{n}(\%)$ & $\begin{array}{l}\text { Dietitians and } \\
\text { doctors, } n(\%)\end{array}$ & Nurses, $n(\%)$ & $\begin{array}{l}\text { Intergroup } \\
p \text {-value* }\end{array}$ \\
\hline Typical timing of enteral feed initiation, $N$ & 113 & 61 & 52 & \\
\hline Within 24 hours of ICU admission & $53(47)$ & $29(47.5)$ & $24(46)$ & \\
\hline Within 48 hours of ICU admission & $25(22)$ & $17(28)$ & $8(15)$ & \\
\hline Within 3 days of ICU admission & $16(14)$ & $13(21)$ & $3(6)$ & \\
\hline Once haemodynamically stable within 5 days & $8(7)$ & $2(3)$ & $6(11.5)$ & \\
\hline Once gastric residual volume below threshold & $1(1)$ & $0(0)$ & $1(2)$ & \\
\hline Depends on individual dietitian/doctor & $10((9)$ & $0(0)$ & $10(19)$ & \\
\hline Most common route, $N$ & 113 & 61 & 52 & \\
\hline Nasogastric & $109(96)$ & $58(95)$ & $51(98)$ & \\
\hline Orogastric & $4(4)$ & $3(5)$ & $1(2)$ & \\
\hline Main rationale for postpyloric feeding, $N$ & 109 & 60 & 49 & 0.020 \\
\hline Only when gastric delivery is unsuccessful & $7(6)$ & $2(3.3)$ & $5(10)$ & \\
\hline Following gastric surgery & $46(42)$ & $19(31)$ & $27(55)$ & \\
\hline In pancreatitis & $9(8)$ & $9(15)$ & $0(0)$ & \\
\hline Hardly ever use it & $45(41)$ & $28(46)$ & $17(35)$ & \\
\hline When gastric emptying delayed for long periods & $2(2)$ & $2(3.3)$ & $0(0)$ & \\
\hline Procedure used for tube insertion, $N$ & 111 & 58 & 53 & \\
\hline Blind, bedside placement by nurses & $6(5)$ & $5(9)$ & $1(2)$ & \\
\hline Blind, bedside placement by dietitian/doctor & $91(82)$ & $43(74)$ & $48(91)$ & \\
\hline Fluoroscopy-assisted & $3(3)$ & $3(5)$ & $0(0)$ & \\
\hline In the operating theatre & $11(10)$ & $7(12)$ & $4(8)$ & \\
\hline Procedure used for checking feeding tube position & 114 & 61 & 53 & \\
\hline Chest X-ray & $45(39)$ & $30(49)$ & $15(19)$ & 0.015 \\
\hline Auscultation of injected air & $26(23)$ & $4(6.6)$ & $22(41.5)$ & 0.000 \\
\hline $\mathrm{pH}$ measurement/litmus paper & $16(14)$ & $3(5)$ & $13(24.5)$ & 0.000 \\
\hline Aspiration of bile-stained fluid & $3(3)$ & $0(0)$ & $3(6)$ & \\
\hline Unsure & $24(21)$ & $24(39)$ & $0(0)$ & \\
\hline Pattern of feed delivery, $N$ & 113 & 61 & 53 & \\
\hline Continuous over 24 hours without any breaks & $52(46)$ & $36(59)$ & $16(30)$ & 0.001 \\
\hline Continuous, with short holds for tolerance check & $45(40)$ & $21(34)$ & $24(45)$ & 0.020 \\
\hline Continuous daytime, stopped during the night & $7(6)$ & $2(3)$ & $5(9)$ & \\
\hline Depends on individual dietitian/doctor & $7(6)$ & $0(0)$ & $7(13)$ & \\
\hline According to judgement of nurse & $3(3)$ & $2(3)$ & $1(2)$ & \\
\hline Frequency of changing feeding tube, $N$ & 112 & 60 & 52 & \\
\hline Only if clinically indicated & $60(54)$ & $26(43)$ & $34(65)$ & 0.010 \\
\hline Routine daily & $14(13)$ & $10(17)$ & $4(7.6)$ & \\
\hline Unsure & $24(21)$ & $24(40)$ & $0(0)$ & \\
\hline According to judgement of nurse & $14(13)$ & $0(0)$ & $14(27)$ & \\
\hline Frequency of changing feed giving set, $N$ & 113 & 61 & 52 & \\
\hline Daily & $72(64)$ & $33(54)$ & $39(75)$ & 0.008 \\
\hline Every time a new feed package is hung up & $18(16)$ & $8(13)$ & $10(19)$ & \\
\hline Randomly (no specific routine) & $5(4)$ & $1(1.6)$ & $4(8)$ & \\
\hline Unsure & $19(17)$ & $19(31)$ & $0(0)$ & \\
\hline Frequency of checking feed tolerance, $N$ & 114 & 61 & 53 & \\
\hline Every 4 - 6 hours throughout ICU stay & $24(21)$ & $24(39)$ & $0(0)$ & 0.000 \\
\hline Every 4 - 6 hours only until feeds established & $58(51)$ & $17(28)$ & $41(77)$ & 0.000 \\
\hline
\end{tabular}




\begin{tabular}{|c|c|c|c|c|}
\hline & Total group, $n(\%)$ & $\begin{array}{l}\text { Dietitians and } \\
\text { doctors, } n(\%)\end{array}$ & Nurses, $n(\%)$ & $\begin{array}{l}\text { Intergroup } \\
p \text {-value* }\end{array}$ \\
\hline Once daily & $24(21)$ & $17(28)$ & $7(13)$ & 0.040 \\
\hline Unsure & $3(3)$ & $3(5)$ & $0(0)$ & \\
\hline No standard - as requested by doctor & $1(1)$ & $0(0)$ & $1(2)$ & \\
\hline Done by doctor/dietitian on ward round & $2(2)$ & $0(0)$ & $2(4)$ & \\
\hline Method of checking feed tolerance, $N$ & 112 & 61 & 51 & \\
\hline Gastric residual volume (GRV) & $61(54)$ & $14(23)$ & $47(92)$ & 0.000 \\
\hline Gastrointestinal (GI) symptoms & $2(2)$ & $2(3)$ & $0(0)$ & \\
\hline Combination of GRV and GI symptoms & $35(31)$ & $35(57)$ & $0(0)$ & 0.000 \\
\hline Combined GRV, Gl symptoms and intra-abdominal pressure monitoring & $10(9)$ & $6(10)$ & $4(8)$ & \\
\hline Unsure & $4(4)$ & $4(7)$ & $0(0)$ & \\
\hline GRV used to guide enteral feeding, $N$ & 40 & 14 & 26 & \\
\hline GRV not used at all to guide practice & $17(43)$ & $14(100)$ & $3(11.5)$ & $<0.050$ \\
\hline No specific threshold applied & $5(13)$ & $0(0)$ & $5(19)$ & \\
\hline Different doctors/dietitians use different volumes & $18(45)$ & $0(0)$ & $18(69)$ & \\
\hline Methods of optimising enteral nutrition, $N$ & 108 & 59 & 49 & \\
\hline Routine use of gastric acid suppression agents & $9(8)$ & $3(5)$ & $6(12)$ & \\
\hline Routine use of prokinetic agents & $17(16)$ & $9(15)$ & $8(16)$ & \\
\hline Use of above medications when indicated & $38(35)$ & $27(46)$ & $11(22)$ & 0.009 \\
\hline Use of above medications on nurse request & $23(21)$ & $7(12)$ & $16(33)$ & 0.008 \\
\hline Change to another enteral feed & $21(19)$ & $13(22)$ & $8(16)$ & \\
\hline Frequency of monitoring enteral nutrition support, $N$ & 105 & 59 & 46 & \\
\hline Daily & $88(84)$ & $56(95)$ & $32(69.5)$ & 0.0005 \\
\hline Only as clinically indicated & $7(6)$ & $2(3.3)$ & $5(11)$ & \\
\hline Randomly & $4(4)$ & $1(2)$ & $3(6.5)$ & \\
\hline Depends on individual dietitian/doctor & $6(6)$ & $0(0)$ & $6(13)$ & \\
\hline Methods of enteral nutrition monitoring, $N$ & 107 & 59 & 48 & \\
\hline Compliance with enteral feeding protocols & $2(2)$ & $1(1.6)$ & $1(2)$ & \\
\hline Compliance with prescribed product & $1(1)$ & $0(0)$ & $1(2)$ & \\
\hline Compliance with prescribed rate & $1(1)$ & $0(0)$ & $1(2)$ & \\
\hline Compliance with nutritional goals & $3(3)$ & $1(1.6)$ & $2(4)$ & \\
\hline Clinical signs/symptoms of intolerance & $5(5)$ & $0(0)$ & $5(10)$ & \\
\hline Combination of the above methods & $88(81)$ & $56(95)$ & $32(67)$ & 0.0005 \\
\hline Unsure & $7(6)$ & $1(1.6)$ & $6(12.5)$ & \\
\hline
\end{tabular}

of parenteral nutrition monitoring were different between the reports of nurses and those of doctors, with $24 \%$ of nurses reporting uncertainty about such practice aspects.

\section{Types of nutrition products used}

Types of enteral products and the frequency of use are presented in Figs 3 and 4 . Of the responses from dietitians and doctors, $49 \%$ stated that no standard starter feed was used. For nurses, $12 \%$ of respondents reported being unsure of the features of different enteral feed types. The most important criteria for enteral product choice were patient tolerance $(97 \%)$, clinical appropriateness of product features (89\%), followed by availability on the hospital pharmacy formulary (38\%). Factors considered unimportant for determining product choice were cost (43\%), pharmacist preference $(73 \%)$ and manufacturer service support (29\%). For the nurses subgroup, $85 \%$ reported that they were unsure of the criteria for choice of enteral and parenteral feed products.
Parenteral products were found to be mainly premixed industry compounded bags (65\%) followed by multichamber bags (30\%), while the remaining respondents reported use of both types of parenteral products. Factors considered most important in parenteral product selection were that nutrition requirements were met (91\%), clinical appropriateness for the patient (84\%) and an appropriate electrolyte profile (78\%). Among nurses (42\%) but not dietitians (14\%), an important factor for parenteral product choice was a diagnosis matched to information on product marketing material. For $47 \%$ of respondents, cost was not an important criterion for parenteral product choice.

\section{Perceptions and self-reports of skill in NSPs}

Dietitians and doctors self-rated their competence in ICU nutrition support as expert (21\%) and above average (70\%). Similarly, nurses' reported perceptions of dietitians' nutrition competence was expert $(61 \%)$ and above average (23\%), while their perception of the skill 


\begin{tabular}{|c|c|c|c|c|}
\hline & $\begin{array}{l}\text { Total group, } \\
n(\%)\end{array}$ & $\begin{array}{l}\text { Dietitians and } \\
\text { doctors, } n(\%)\end{array}$ & Nurses, $n(\%)$ & $\begin{array}{l}\text { Intergroup } \\
p \text {-value* }\end{array}$ \\
\hline Typical timing of parenteral nutrition initiation, $N$ & 100 & 53 & 47 & \\
\hline Within 24 hours of ICU admission & $20(20)$ & $8(15)$ & $12(26)$ & \\
\hline As soon as clinically indicated regardless of timing & $30(30)$ & $16(30)$ & $14(30)$ & \\
\hline Within 3 days if enteral feeding unsuccessful & $7(7)$ & $3(6)$ & $4(8.5)$ & \\
\hline After 7 days if enteral feeding unsuccessful & $3(3)$ & $3(6)$ & $0(0)$ & \\
\hline Immediately in malnourished patients & $7(7)$ & $3(6)$ & $4(8.5)$ & \\
\hline When clinically indicated $>3$ days of unsuccessful enteral & $19(19)$ & $14(26)$ & $5(11)$ & \\
\hline When clinically indicated $>7$ days of unsuccessful enteral & $4(4)$ & $4(7.5)$ & $0(0)$ & \\
\hline When clinically indicated in malnourished patients & $2(2)$ & $2(4)$ & $0(0)$ & \\
\hline Depends on dietitian/doctor & $8(8)$ & $0(0)$ & $8(17)$ & \\
\hline Usual rationale for parenteral nutrition, $N$ & 100 & 54 & 46 & \\
\hline Provide requirements only when enteral nutrition not possible & $75(75)$ & $47(87)$ & $28(61)$ & 0.0065 \\
\hline Provide requirements in all ICU patients & $8(8)$ & $3(5.5)$ & $5(11)$ & \\
\hline Supplement enteral nutrition in all malnourished patients & $1(1)$ & $0(0)$ & $1(2)$ & \\
\hline Supplement enteral when requirements not met & $12(12)$ & $3(5.5)$ & $9(19.5)$ & 0.0300 \\
\hline Routine to start with parenteral in GIT surgery & $4(4)$ & $1(2)$ & $3(6.5)$ & \\
\hline Typical duration of parenteral nutrition, $N$ & 101 & 54 & 47 & \\
\hline $0-5$ days & $53(53)$ & $26(48)$ & $27(57)$ & \\
\hline 7 days & $31(31)$ & $20(37)$ & $11(23)$ & \\
\hline$>7$ days & $16(16)$ & $8(15)$ & $8(17)$ & \\
\hline$>14$ days & $1(1)$ & $0(0)$ & $1(2)$ & \\
\hline Frequency of monitoring of parenteral nutrition, $N$ & 100 & 54 & 46 & \\
\hline Daily & $60(60)$ & $38(70)$ & $22(48)$ & 0.0250 \\
\hline 1 - 3 times weekly & $18(18)$ & $13(24)$ & $5(11)$ & \\
\hline Randomly, as clinically indicated & $10(10)$ & $2(4)$ & $8(17)$ & \\
\hline Unsure & $12(12)$ & $1(2)$ & $11(24)$ & 0.0010 \\
\hline Methods of parenteral nutrition monitoring, $N$ & 100 & 53 & 47 & \\
\hline Compliance with parenteral feeding protocol & $2(2)$ & $0(0)$ & $2(4)$ & \\
\hline Compliance with prescribed product & $1(1)$ & $0(0)$ & $1(2)$ & \\
\hline Compliance with prescribed rate & $2(2)$ & $0(0)$ & $2(4)$ & \\
\hline Compliance with nutritional goals & $3(3)$ & $0(0)$ & $3(6)$ & \\
\hline Clinical signs/symptoms of intolerance & $1(1)$ & $1(2)$ & $0(0)$ & \\
\hline Combination of above methods & $82(82)$ & $51(96)$ & $31(66)$ & 0.0005 \\
\hline Unsure & $9(9)$ & $1(2)$ & $8(17)$ & \\
\hline
\end{tabular}

of doctors in this discipline was expert (19\%) and above average (43\%). However, $38 \%$ of nurses rated doctors working in their units as below average or totally unskilled in nutrition support of critically ill patients. Nurses'self-report of their own skills indicated mainly average/ satisfactory (40\%) and above average (47\%) competence.

\section{Discussion}

This is the first comprehensive survey of ICU nutrition practices to be performed in SA. The data from this pilot study showed a high selfconfidence in self-reported nutrition support competency among dietitians working in private ICUs, matched by a perception from nursing staff that dietitians are expertly skilled in this practice. In contrast, nurses perceived their own nutritional skill to be lower than that of dietitians, but also reported a considerable lack of perceived nutrition support competency among doctors working in critical care units. These varied competency levels may be problematic for the integrated delivery of nutrition support in ICU.

Critical care nutrition is not a core entry-level skill for health professionals in SA and there is no regulatory requirement for postbasic qualification or certification. Varied levels of practitioner expertise between healthcare professionals qualified in different disciplines emphasise the need for good multidisciplinary cooperation in order to achieve nutrition support goals, and positively affect clinical outcomes. In the context of these perceived skill disparities among various healthcare professionals, less than half of the ICUs surveyed reported the operation of a formal nutrition support team within the unit. There was also a significant discrepancy between reports by nurses and dietitians in this regard, with nurses being significantly more likely 


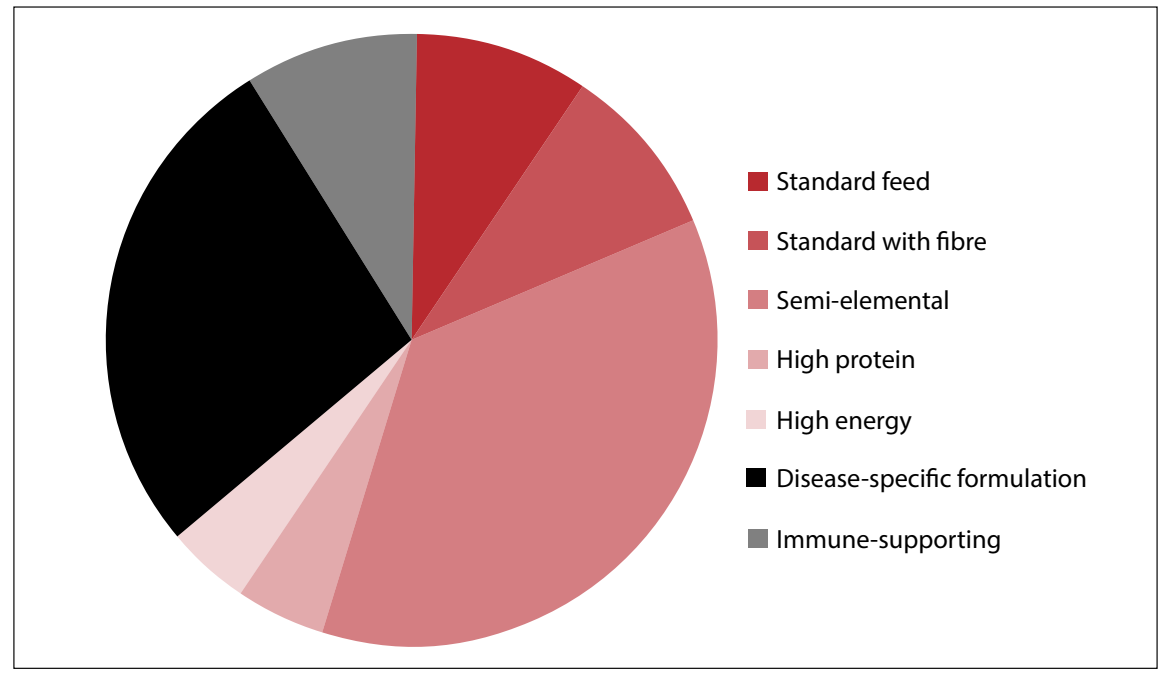

Fig. 3. Types of enteral formulas administered.

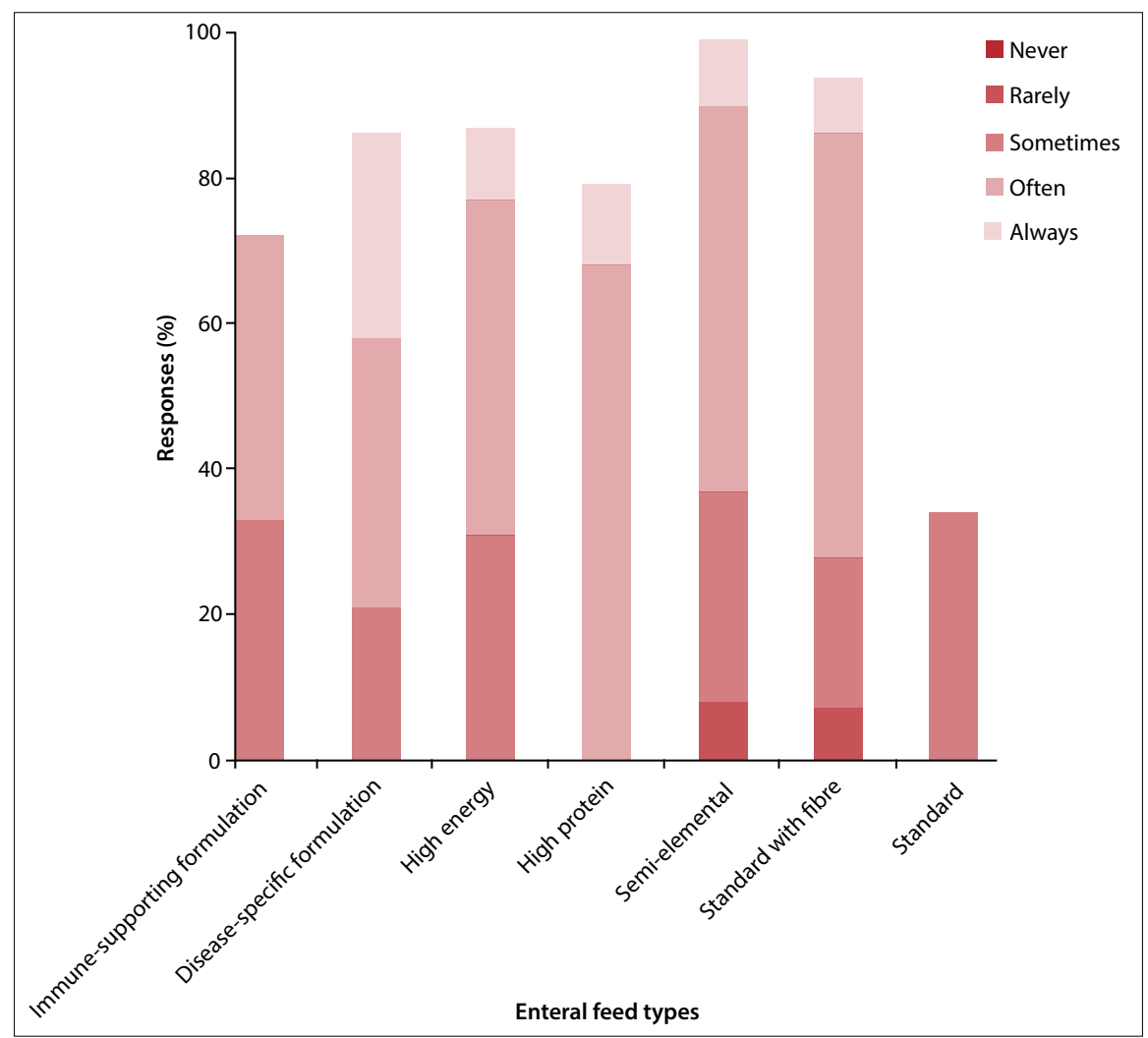

Fig. 4. Frequency of use of enteral formulations.

than dietitians to report the presence of a nutrition support team, yet nurse inclusion in such a team was low, as was nurse decision-making involvement in nutrition support. Team structure and composition varied between units, but reports indicated overall that in one-third of cases the dietitian was the only member of the team, and less than half of the team compositions included nursing staff. Furthermore, discrepant responses from dietitians and nurses with regards to the practicalities of NSP, including tube management, delivery patterns and monitoring patterns, as well as reports from both subgroups of uncertainty around such with nursing guidelines. ${ }^{[13]}$ Nutrition practice guidelines are often not tailored to nursespecific barriers to nutrition delivery, and this is exacerbated by a lack of multidisciplinary collaboration and dietetic input that supports rather than criticises nursing constraints, particularly after working hours. ${ }^{[2]}$ For example, in this survey dietitians and doctors reported a high adherence to published guidelines in ICU NSP, while few nursing staff seemed aware of such guidelines and were more likely to understand (or possibly have observed) nutrition support decision-making as emanating from the clinical judgements of dietitians or doctors. Additionally, nurses seemed to have low involvement in important aspects of feed implementation, such as tube feed placement, while reporting a reliance on their own judgement for various aspects of nutrition support management. Furthermore, differences were seen in nurses' reported practices and dietitians' perceptions of nursing practices. Dietitian and nurse subgroups both reported that they were unaware or unsure of what the other was doing with regard to certain aspects of nutrition support. This does not appear consistent with team management of ICU nutritional care, and does not suggest optimal enabling of nurses to implement nutrition support at a high standard.

One strategy to foster quality integration of nutrition support in ICUs is the use of formal, written nutrition support protocols. Large-scale cohort studies $^{[14]}$ have shown that ICUs that use nutrition protocols have superior nutrition adequacy in terms of delivery of requirements, success of enteral nutrition, achievement of early enteral feeding and methods to optimise enteral nutrition. These SA pilot survey data revealed that approximately two-thirds of ICUs utilised formal, written nutrition support protocols, of which only about half governed both enteral and parenteral nutrition support. Despite reported use of formal protocols and the reported compliance with published nutrition support guidelines, there was a high reported reliance on clinical judgement to guide practice. There was also was a disjuncture between aspects of nutrition practice (rationale, initiation, delivery and monitoring) as reported by dietitians and by nurses. In addition, practice inconsistencies revealed in other sections of the survey instrument suggest that the reported use of nutrition support protocols may not hold true in practice. Importantly, nurse reports indicated that NSPs varied with different individual dietitians and doctors working in the ICU, suggesting that an overarching, protocolised approach to nutrition support did not occur in units. 
For both enteral and parenteral nutrition practices, compliance with recommendations varied in how well they matched with currently available guidelines. For enteral nutrition, initiation decisions and feed delivery pattern generally matched the guidelines, and most units reported adherence to timing of early enteral nutrition given haemodynamic stability of the patient. ${ }^{[1,15]}$ However, rather a high importance was placed on proof of gut function, which may be inappropriate in the critically ill and is not supported by published recommendations. ${ }^{[1]}$ There was a very low utilisation of postpyloric feeding even when enteral feeding was unsuccessful due to extended delays in gastric emptying. This is unfortunate, since postpyloric feeding is a specific strategy to optimise enteral feeding success. ${ }^{[1,15,16]}$ Data also showed excessive use of semi-elemental formulae and very low utilisation of standard formulations compared with international usage data, ${ }^{[9]}$ and also in contradiction to enteral feed guidelines. ${ }^{[15]}$ The nursing subgroup had an undue reliance on gastric residual volumes as a method of checking feed tolerance, unsupported by the literature, ${ }^{[1]}$ and there was a low compliance with methods to optimise enteral feeding delivery. This is in line with studies that have previously shown that optimisation of enteral nutrition support is generally more poorly performed than other aspects of nutrition support, such as glucose control or elevation of the head of the bed..$^{[2,16]}$

For parenteral nutrition practices, there was both a high reported reference to published guidelines and use of clinical judgement. Overall rationale for parenteral use was largely appropriate, but the main determinant of parenteral nutrition initiation was reported to be the presence of gastrointestinal factors likely to make enteral feeding unsuccessful. In contrast, only $7 \%$ indicated this factor to be actually unsuccessful enteral feeding. This indicates that parenteral nutrition initiation was determined by the anticipation of lack of success with enteral feeding rather than proven failure of this route of delivery, in contrast to recommendations. ${ }^{[1]}$ Reported utilisation of parenteral nutrition for malnourished patients appeared low. It is unclear whether this is reflective of a lower adherence to parenteral nutrition guidelines, ${ }^{[1,15,17]}$ or of the adequate nutritional status of ICU patients in private care facilities.

These survey data have limitations. Despite targeted sampling of $>500$ medical doctors known to work in ICUs and coverage of $>60 \%$ of private hospitals with ICU facilities, the overall response from individual healthcare professionals working in ICUs was low. In particular, the response from intensivists and other medical specialists was confined to just $4 \%$ of the entire sample, suggesting that nutrition may not be a priority feature of critical care management for doctors working in ICU. Given that more than a third of nurses rated the doctors working in their units as unskilled in NSPs, this perhaps is not a surprising result. However, it is impossible to comment on the reasons for the overall low response, or comment on the level of selection bias that may exist within the sample. There was also an overall reluctance among participants to share nutrition protocols with the researcher; therefore, this survey could not test participant responses against unit policies and standardised clinical practices that may have been in existence, or even verify the existence of such protocols as reported. In light of these study shortcomings, this research should be viewed as a pilot survey. A more comprehensive survey - or indeed a more robust study design - might produce different results.

Nevertheless, taken together, the data from this survey do raise questions as to the organisation and operations of formal nutrition teams and the implementation of formal nutrition protocols in private ICUs in SA. Inconsistent responses from the professional subgroups represented suggest that such structures may run less formally and effectively than may be believed or intended by the participants. There are several possible implications of this information for the critical care nutrition discipline in SA. It may be true that there are barriers to the implementation of international guidelines in the SA context. If this is the case, then more comprehensive cross-sectional data from a more fully representative sample of prescribers and implementers of nutrition support in ICUs are required. This will allow the experience and input of SA healthcare practitioners to shape national nutrition practices and develop SA nutrition practice guidelines, which are appropriate to any unique demographic or resource-related factors affecting nutrition delivery in this setting. Nevertheless, international data have shown that improved multidisciplinary team approaches translate into improved quality of care. Because of the demonstrated perception that dietitians have nutrition skill not matched by that of other health professionals, there is an opportunity to enhance the positioning of the dietitian as the nutrition expert in ICUs, and build a more holistic team approach to nutritional care of the critically ill patient. There may be a need for a regulatory mechanism for practitioners to prove postbasic competency in critical care nutrition support. Finally, there is a need for improved knowledge-to-practice translation for all disciplines of healthcare professionals involved with nutritional care of the critically ill patient.

Acknowledgements. This research was made possible with financial aid received from Nutricia, SA and Nutricia, Middle East. The author wishes to acknowledge the scientific input of Kuba Morris, Scientific Officer of Nutricia Middle East, South-East Asia and Africa. The author also wishes to gratefully acknowledge the input of all the respondents to this survey

Conflict of interest. The author is a consultant to industry and has consulted to Nutricia, SA and BBraun, SA.

References

1. McClave SA, Martindale RG, Vanek VW, et al; ASPEN Board of Directors; American College of Critical Care Medicine; Society of Critical Care Medicine. Guidelines for the provision and assessment of nutrition support therapy in the adult critically ill patient: Society of Critical Care Medicine (SCCM) and American Society for Parenteral and Enteral Nutrition (ASPEN). JPEN
Parenter Enteral Nutr 2009;33(3):277-316. [http://dx.doi.org/10.1177/0148607109335234]

2. Cahill NE, Murch L, Cook D, Heyland DK on behalf of the Canadian Critical Care Trials Group. Improving the provision of enteral nutrition in the intensive care unit: A description of a multifaceted intervention tailored to overcome local barriers. Nutr Clin Pract 2014;29(1):110117. [http://dx.doi.org/10.1177/0884533613516512]

3. Farber M, Moses J, Korn M. Reducing costs and patient morbidity in the enterally fed intensive care unit patient. JPEN J Parenter Enteral Nutr 2005;29(1 Suppl):S62e9. [http://dx.doi.org/10.11 77/01486071050290s1s62]

4. Heyland D, Schroter-Noppe D, Drover J, et al. Nutrition support in the critical care setting: Current practice in Canadian ICUs - opportunities for improvement? JPEN J Parenter Entera Nutr 2003;27(1):74e83. [http://dx.doi.org/10.1177/014860710302700174]

5. Thomas DR. Starving in the hospital. Nutrition 2003;19(10):907e8. [http://dx.doi.org/10.1016/ s0899-9007(03)00169-2]

6. Villet S, Chiolero R, Bollmann M, et al. Negative impact of hypocaloric feeding and energy balance on clinical outcome in ICU patients. Clin Nutr 2005;24(4):502e9. [http://dx.doi.org/10.1016/j. clnu.2005.03.006]

7. Sheean PM, Peterson SJ, Gurka DP, Braunschweig CA. Nutrition assessment: The reproducibility Sheean PM, Peterson SJ, Gurka DP, Braunschweig CA. Nutrition assessment: The reproducibility
of Subjective Global Assessment in patients requiring mechanical ventilation. Eur J Clin Nutr 2010;64(11):1358-1364. [http://dx.doi.org/10.1038/ejen.2010.154]

8. Shpata V, Prendushi X, Kreka M, Kola I, Kurti F, Ohri I. Malnutrition at the time of surgery affects negatively the clinical outcome of critically ill patients with gastrointestinal cancer. Med Arch 2014;68(4):263-267. [http://dx.doi.org/10.5455/medarh.2014.68.263-267]

9. Roynette $C E$, Bongers A, Fulbrook P, Albarran JW, Hofman Z. Enteral feeding practices in European ICUs: A survey from the European federation of critical care nursing associations (EfCCNa). E Spen Eur E J Clin Nutr Metab 2008;3:e33ee39. [http://dx.doi.org/10.1016/j.eclnm.2007.10.004]

10. Scribante J, Bhagwanjee S. National audit of critical care resources in South Africa - open versus closed intensive and high care units. S Afr Med J 2007:97(12):1319-1322.

11. Hodgson RE. The management of death in the ICU. The South African perspective. Crit Care Resusc 2006;8:73-75.

12. Mathivha LR. ICUs worldwide: An overview of critical care medicine in South Africa. J Crit Care 2002;6(1):22-23. [http://dx.doi.org/10.1186/cc1449]

13. Marshall AP, Cahill N, Gramlich L, MacDonald G, Alberda C, Heyland DK. Optimising nutrition in intensive care units: Empowering critical care nurses to be effective agents of change. Am J in intensive care units: Empowering critical care nurses to be effective
Crit Care 2012;21(3):186-194. [http://dx.doi.org/10.4037/ajcc2012697]

14. Heyland DK, Cahill N, Dhaliwal R, Sun X, Day AG, McClave SA. Impact of enteral feeding protocols on enteral nutrition delivery: Results of a multicenter observational study. JPEN J Parenter Enteral Nutr 2010;34(6):675-684. [http://dx.doi.org/10.1177/0148607110364843]

15. Kreymann KG, Berger MM, Deutz N, et al. ESPEN Guidelines on Enteral Nutrition: Intensive care. Clin Nutr 2006;25(2):210-223. [http://dx.doi.org/10.1016/j.clnu.2006.01.021]

16. Dhaliwal R, Cahill N, Lemieux M, Heyland DK. The Canadian critical care nutrition guidelines in 2013: An update on current recommendations and implementation strategies. Nutr Clin Pract 2014;29(1):29-43. [http://dx.doi.org/10.1177/0884533613510948.6]

17. Singer $P$, Berger $M M$, van den Berghe G, et al. ESPEN Guidelines on Parenteral Nutrition: Intensive care. Clinical Nutrition 2009;28(4):387-400. [ttp://dx.doi.org/10.1016/j.clnu.2009.04.024] 